\title{
On the Admissibility of Singular Perturbations in Cauchy Problems II
}

By

\author{
Ryuichi AsHINo*
}

\begin{abstract}
We shall give a new proof of the admissibility of singular perturbations, which was introduced by the author [2], without the assumption on the characteristic roots assumed in [2] using the method introduced by the author in [5, Appendix].
\end{abstract}

\section{$\S 1$. Introduction}

Let us consider the following linear partial differential operator of kowalewskian type with constant coefficients containing small positive parameter $\varepsilon$ satisfying $0 \leq \varepsilon<1$ :

$$
L_{\varepsilon}(D)=\varepsilon \cdot P_{1}(D)+P_{2}(D)
$$

Denote by $m$ the order of $P_{1}(D)$ with respect to $D_{1}$ and by $m^{\prime}$ that of $P_{2}(D)$. Put $m^{\prime \prime}=m-m^{\prime}$ and assume that $m>m^{\prime}>0$. Then the order of $L_{0}$ is less than that of $L_{\varepsilon}$ for $\varepsilon \neq 0$. Such an operator as $L_{\varepsilon}$ is called a singularly perturbed operator.

We shall study the following so-called singularly perturbed unilateral Cauchy problems for $L_{\varepsilon}(D)$ in $\boldsymbol{R}_{+}^{n}=\left\{x \in \boldsymbol{R}^{n} ; x_{1}>0\right\}$ :

$$
\left\{\begin{array}{l}
L_{\varepsilon}(D) u(x)=0, \quad \text { in } R_{+}^{n} ; \\
\lim _{\delta \downarrow 0} D_{1}^{j-1} u\left(\delta, x^{\prime}\right)=\varphi_{j}\left(x^{\prime}\right), \quad j=1, \cdots, m,
\end{array}\right.
$$

and the following so-called reduced unilateral Cauchy problem for (CP):

Communicated by T. Kawai, July 27, 1993. Revised September 30, 1993 and March 7, 1994. 1991 Mathematics Subject Classification: 35B25

* College of Industrial Technology, 1-27, Nishikoya, Amagasaki, 661, Japan. 
$(\mathrm{RCP})$

$$
\left\{\begin{array}{l}
L_{0}(D) u(x)=0, \quad \text { in } \mathbb{R}_{+}^{n} ; \\
\lim _{\delta \downarrow 0} D_{1}^{j-1} u\left(\delta, x^{\prime}\right)=\varphi_{j}\left(x^{\prime}\right), \quad j=1, \cdots, m^{\prime} .
\end{array}\right.
$$

Put $\varphi^{\prime}=\left(\varphi_{1}, \cdots, \varphi_{m^{\prime}}\right), \varphi^{\prime \prime}=\left(\varphi_{m^{\prime}+1}, \cdots, \varphi_{m}\right)$, and $\varphi=\left(\varphi^{\prime}, \varphi^{\prime \prime}\right)$. Denote by $A^{\prime}$ the space of Cauchy data $\varphi^{\prime}$, by $A^{\prime \prime}$ the space of Cauchy data $\varphi^{\prime \prime}$, and by $A=A^{\prime} \times A^{\prime \prime}$ the space of Cauchy data $\varphi$, respectively. Hereafter, we assume that (CP) is uniquely solvable in $C\left(\mathbb{R}_{+}^{n}\right)$ for every $\varphi \in A$ and (RCP) is uniquely solvable in $C\left(\mathbb{R}_{+}^{n}\right)$ for every $\varphi^{\prime} \in A^{\prime}$. For example, if $A=O\left(\mathbb{C}^{n-1}\right)^{m}$, where we denote by $O\left(\mathbb{C}^{n-1}\right)$ the set of entire functions defined in $\mathbb{C}^{n-1}$, then the Cauchy-Kowalewski theorem implies that the Cauchy problems (CP) are globally uniquely solvable. If $A=F^{-1}\left(C_{0}^{\infty}\left(\mathbb{R}^{n-1}\right)\right)^{m}$, where $F^{-1}$ denotes the inverse Fourier transformation, then the Cauchy problems (CP) can be solved uniquely by the Fourier transformation.

Befinition 1.1. The unilateral Cauchy problems (CP) with the Cauchy data space $A$ are said to be admissible with respect to the reduced unilateral Cauchy problem (RCP) with the Cauchy data space $A^{\prime}$ if every Cauchy data $\varphi$ in $A$, the solutions $u_{\varepsilon}(x ; \varphi)$ of $(\mathrm{CP})$ converge to the solution $u_{0}\left(x ; \varphi^{\prime}\right)$ of $(\mathrm{RCP})$ in $C\left(\mathbb{R}_{+}^{n}\right)$.

Let the symbols of $P_{1}(D)$ and $P_{2}(D)$ be represented as

$$
\begin{aligned}
& P_{1}(\xi)=\sum_{j=0}^{m} p_{1, j}\left(\xi^{\prime}\right) \xi_{1}^{m-j} \\
& P_{2}(\xi)=\sum_{j=0}^{m^{\prime}} p_{2, j}\left(\xi^{\prime}\right) \xi_{1}^{m^{\prime}-j}
\end{aligned}
$$

where $p_{1.0}$ and $p_{2,0}$ are non-zero constants. Put $p=p_{2,0} / p_{1,0}$. The following theorem has already been stated in [2] under the assumption that for the characteristic roots of $P_{2}(\xi)=0$ with respect to $\xi_{1}$, which we denote $\sigma_{j}\left(\xi^{\prime}\right)$, $j=1, \cdots, m^{\prime}$, there exists a point $\xi_{0}^{\prime}$ in $\mathbb{R}^{n-1}$ such that

$$
\sigma_{j}\left(\xi_{0}^{\prime}\right) \neq \sigma_{k}\left(\xi_{0}^{\prime}\right), \quad 1 \leq j<k \leq m^{\prime} .
$$

But using a new expansion formula of a certain meromorphic function defined as the quotient of two determinants, which will be stated in Proposition, we can remove such an assumption as the above. This formula is proved by calculating the Laplace expansions of these determinants, which was essentially 
stated in [5, Appendix]. Our aim in this paper is to give a new proof of Theorem (based on this expansion formula).

For a complex number $p$, we denote by $\Im p$ the imaginary part of $p$ and by $\mathfrak{R} p$ the real part of $p$.

Theorem. The unilateral Cauchy problems $(C P)$ with $A=F^{-1}\left(C_{0}^{\infty}\left(R^{n-1}\right)\right)^{m}$ are asmissible with respect to $(R C P)$ if and only if either the following conditions (C1) or (C2) is satisfied.

$$
\begin{aligned}
& m^{\prime \prime}=2 \text { and } p<0 ; \\
& m^{\prime \prime}=1 \text { and } \Im p \leq 0 .
\end{aligned}
$$

\section{$\S 2 . \quad$ Preliminaries}

We shall state here notation and lemmas without proofs. The proofs are essentially the same as [5, Appendix]. We start with introducing general notations.

Let $n$ be an integer. We shall substitute $n$ for $m, m^{\prime}, m^{\prime \prime}$, and so on. Let $z=\left(z_{1}, \cdots, z_{n}\right)$ and $w=\left(w_{1}, \cdots, w_{n}\right)$ be complex variables. For a non-negative integer $l$, denote $a(l)(z)=\left(\left(z_{j}\right)^{l} ; j \rightarrow 1, \cdots, n\right)$ and for non-negative integers $l_{1}, \cdots, l_{n}$ satisfying $0 \leq l_{1}<\cdots<l_{n}$, denote

$$
A\left(l_{1}, \cdots, l_{n}\right)(z)=\operatorname{det}\left(a\left(l_{i}\right)(z) ; i \downarrow 1, \cdots, n\right) .
$$

Let $i=\sqrt{-1}$ and $x_{1}$ be a real parameter. Denote $e\left(w, x_{1}\right)=\left(e^{i w_{j} x_{1}} ; j \rightarrow 1, \cdots, n\right)$ and for non-negative integers $l_{1}, \cdots, l_{n-1}$ satisfying $0 \leq l_{1}<\cdots<l_{n-1}$, denote

$$
B\left(l_{1}, \cdots, l_{n-1}\right)\left(z, w, x_{1}\right)=\operatorname{det}^{t}\left({ }^{t} e\left(w, x_{1}\right),{ }^{t} a\left(l_{1}\right)(z), \cdots,{ }^{t} a\left(l_{n-1}\right)(z)\right) .
$$

Expand the determinant $B\left(l_{1}, \cdots, l_{n-1}\right)\left(z, w, x_{1}\right)$ with respect to the first row. Then

$$
B\left(l_{1}, \cdots, l_{n-1}\right)\left(z, w, x_{1}\right)=\sum_{j=1}^{n}(-1)^{1+j} A\left(l_{1}, \cdots, l_{n-1}\right)(z(j)) e^{i w_{j} x_{1}},
$$

where $z(j)=\left(z_{1}, \cdots, z_{j-1}, z_{j+1}, \cdots, z_{n}\right)$. Denote

$$
C\left(l_{1}, \cdots, l_{n}\right)(z)=A\left(l_{1}, \cdots, l_{n}\right)(z) / A(0, \cdots, n-1)(z)
$$

and

$$
D\left(l_{1}, \cdots, l_{n-1}\right)\left(z, w, x_{1}\right)=B\left(l_{1}, \cdots, l_{n-1}\right)\left(z, w, x_{1}\right) / A(0, \cdots, n-1)(z) .
$$


Then $C\left(l_{1}, \cdots, l_{n}\right)(z)$ is a homogeneous symmetric polynomial in $Z[z]$ of order $l_{1}+\cdots+l_{n}-(n-1) n / 2$ and $D\left(l_{1}, \cdots, l_{n-1}\right)\left(z, w, x_{1}\right)$ is meromorphic in $z$ and entire in $w$. Put

$$
E_{j}(z)=\left\{(-1)^{n-j} \prod_{k \neq j, 1 \leq k \leq n}\left(z_{j}-z_{k}\right)\right\}^{-1}, \quad j=1, \cdots, n
$$

Then $E_{j}(z), j=1, \cdots, n$ are meromorphic in $C^{n}$ and holomorphic when $z_{i} \neq z_{j}$, $1 \leq i<j \leq n$. As a meromorphic function in $z$, we have

$$
\begin{gathered}
D\left(l_{1}, \cdots, l_{n-1}\right)\left(z, w, x_{1}\right) \\
=\sum_{j=1}^{n}(-1)^{1+j} C\left(l_{1}, \cdots, l_{n-1}\right)(z(j)) e^{i w_{j} x_{1}} E_{j}(z) .
\end{gathered}
$$

Let $m, m^{\prime}$, and $m^{\prime \prime}$ be positive integers such that $m=m^{\prime}+m^{\prime \prime}$. Denote $z^{\prime}=\left(z_{1}, \cdots, z_{m^{\prime}}\right), z^{\prime \prime}=\left(z_{m^{\prime}+1}, \cdots, z_{m}\right)$, and $z=\left(z^{\prime}, z^{\prime \prime}\right)$. Denote $w=\left(w^{\prime}, w^{\prime \prime}\right)$ in the same manner. Let $l_{1}, \cdots, l_{m-1}$ be non-negative integers satisfying $0 \leq l_{1}<\cdots<$ $l_{m-1}$. Let $S_{1}$ be the set of all bijections $\rho$ from $\{1, \cdots, m-1\}$ onto $\left\{l_{1}, \cdots, l_{m-1}\right\}$ satisfying

$$
\rho(1)<\cdots<\rho\left(m^{\prime}\right) ; \rho\left(m^{\prime}+1\right)<\cdots<\rho(m-1)
$$

and $S_{2}$ be the set of all bijections $\rho$ from $\{1, \cdots, m-1\}$ onto $\left\{l_{1}, \cdots, l_{m-1}\right\}$ satisfying

$$
\rho(1)<\cdots<\rho\left(m^{\prime}-1\right) ; \rho\left(m^{\prime}\right)<\cdots<\rho(m-1) \text {. }
$$

Each bijection in $S_{1}$ uniquely corresponds to a way of selecting $m^{\prime}$ objects from $m-1$ objects and each bijection in $S_{2}$ uniquely corresponds to a way of selecting $m^{\prime}-1$ objects from $m-1$ objects, respectively. Define the bijection $\pi$ from $\left\{l_{1}, \cdots, l_{m-1}\right\}$ onto $\{2, \cdots, m\}$ as $\pi\left(l_{j}\right)=j+1, j=1, \cdots, m-1$. Denote

$$
I(\rho)=\sum_{j=1}^{m^{\prime}} \pi(\rho(j))+m^{\prime}\left(m^{\prime}+1\right) / 2 ; \quad J(\rho)=1+\sum_{j=1}^{m^{\prime}-1} \pi(\rho(j))+m^{\prime}\left(m^{\prime}+1\right) / 2 .
$$

For $z_{i} \neq z_{j}, 1 \leq i \leq m^{\prime}, m^{\prime}+1 \leq j \leq m$, denote

$$
E(z)=\prod_{1 \leq i \leq m^{\prime}, m^{\prime}+1 \leq j \leq m}\left(z_{j}-z_{i}\right)^{-1}
$$




$$
\begin{aligned}
& D_{1}\left(l_{1}, \cdots, l_{m-1}\right)\left(z, w, x_{1}\right) \\
& =\sum_{\rho \in S_{1}}(-1)^{I(\rho)} C\left(\rho(1), \cdots, \rho\left(m^{\prime}\right)\right)\left(z^{\prime}\right) \\
& \quad \times D\left(\rho\left(m^{\prime}+1\right), \cdots, \rho(m-1)\right)\left(z^{\prime \prime}, w^{\prime \prime}, x_{1}\right) E(z) ; \\
& D_{2}\left(l_{1}, \cdots, l_{m-1}\right)\left(z, w, x_{1}\right) \\
& =\sum_{\rho \in S_{2}}(-1)^{J(\rho)} D\left(\rho(1), \cdots, \rho\left(m^{\prime}-1\right)\right)\left(z^{\prime}, w^{\prime}, x_{1}\right) \\
& \quad \times C\left(\rho\left(m^{\prime}\right), \cdots, \rho(m-1)\right)\left(z^{\prime \prime}\right) E(z) .
\end{aligned}
$$

Then we have the following new expansion formula. As the proof is similar as that of [5, Lemma A.1], we omit it.

Proposition. For $z_{i} \neq z_{j}, 1 \leq i \leq m^{\prime}, m^{\prime}+1 \leq j \leq m$,

$$
\begin{aligned}
& D\left(l_{1}, \cdots, l_{m-1}\right)\left(z, w, x_{1}\right) \\
& \quad=D_{1}\left(l_{1}, \cdots, l_{m-1}\right)\left(z, w, x_{1}\right)+D_{2}\left(l_{1}, \cdots, l_{m-1}\right)\left(z, w, x_{1}\right) .
\end{aligned}
$$

Hereafter in this section, we assume that $\left\{l_{1}, \cdots, l_{m-1}\right\}=\{0, \cdots, k-1, k+1, \cdots$, $m-1\}$. For $k=m^{\prime}, \cdots, m-1$, for $j=1, \cdots, m^{\prime \prime}$, and for $\rho \in S_{1}$, denote

$$
\begin{gathered}
z^{\prime \prime}(j)=\left(z_{m^{\prime}+1}, \cdots, z_{m^{\prime}+j-1}, z_{m^{\prime}+j+1}, \cdots, z_{m}\right) ; \\
E_{j}\left(z^{\prime \prime}\right)=\left\{(-1)^{m^{\prime \prime}+j} \prod_{k \neq j, 1 \leq k \leq m^{\prime \prime}}\left(z_{m^{\prime}+j}-z_{m^{\prime}+k}\right)\right\}^{-1} ; \\
f_{k, j, \rho}(z)=(-1)^{I(\rho)+1+j} C\left(\rho(1), \cdots, \rho\left(m^{\prime}\right)\right)\left(z^{\prime}\right) \\
\times C\left(\rho\left(m^{\prime}+1\right), \cdots, \rho(m-1)\right)\left(z^{\prime \prime}(j)\right) E_{j}\left(z^{\prime \prime}\right) E(z) ; \\
F_{k, j}(z)=\sum_{\rho \in S_{1}} f_{k, j, \rho}(z) .
\end{gathered}
$$

These $F_{k, j}$ are rational functions in $z$ and holomorphic if $z_{i} \neq z_{j}$ are satisfied for $1 \leq i \leq m^{\prime}$ and $m^{\prime}+1 \leq j \leq m$ and for $m^{\prime}+1 \leq i<j \leq m$. 
Lemma 2.1. Assume that $z_{i} \neq z_{j}$ are satisfied for $1 \leq i \leq m^{\prime}$ and $m^{\prime}+1 \leq j \leq m$ and for $m^{\prime}+1 \leq i<j \leq m$. Then, for $k=m^{\prime}, \cdots, m-1$,

$$
D_{1}(0, \cdots, k-1, k+1, \cdots, m-1)\left(z, w, x_{1}\right)=\sum_{j=1}^{m^{\prime \prime}} F_{k, j}(z) e^{i w_{m^{\prime}+\jmath} x_{1}} .
$$

Proof. By (2.1), we have

$$
\begin{gathered}
D\left(\rho\left(m^{\prime}+1\right), \cdots, \rho(m-1)\right)\left(z^{\prime \prime}, w^{\prime \prime}, x_{1}\right) \\
=\sum_{j=1}^{m^{\prime \prime}}(-1)^{1+j} C\left(\rho\left(m^{\prime}+1\right), \cdots, \rho(m-1)\right)\left(z^{\prime \prime}(j)\right) e^{i w_{m^{\prime}+j} x_{1}} E_{j}\left(z^{\prime \prime}\right) .
\end{gathered}
$$

On the other hand, by the definition, we have

$$
\begin{aligned}
& D_{1}(0, \cdots, k-1, k+1, \cdots, m-1)\left(z, w, x_{1}\right) \\
= & \sum_{\rho \in S_{1}} C\left(\rho(1), \cdots, \rho\left(m^{\prime}\right)\right)\left(z^{\prime}\right) \\
& \times D\left(\rho\left(m^{\prime}+1\right), \cdots, \rho(m-1)\right)\left(z^{\prime \prime}, w^{\prime \prime}, x_{1}\right) E(z) .
\end{aligned}
$$

Substitute (2.2) for (2.3), and we come to the conclusion.

For a positive parameter $\eta$, put $t_{j}=\eta z_{j}, j=1, \cdots, m$. Denote $t^{\prime}=\eta z^{\prime}, t^{\prime \prime}=\eta z^{\prime \prime}$, and $t=\eta z$.

Assumption 2.1. There exists positive numbers $M, M^{\prime}, c$, and $\eta_{0}$ with $\eta_{0} \leq 1$ such that for every $\eta$ satisfying $0<\eta \leq \eta_{0}$, the following estimates are satisfied:

$$
\left|z^{\prime}\right| \leq M ; \quad\left|t^{\prime \prime}\right| \leq M ; \quad \inf _{m^{\prime}+1 \leq i<j \leq m}\left|t_{i}-t_{j}\right| \geq c ; \quad \inf _{1 \leq i \leq m^{\prime}, m^{\prime}+1 \leq j \leq m}\left|t_{i}-t_{j}\right| \geq c .
$$

Denote

$$
M^{\prime}=M^{\prime}\left(w, x_{1}\right)=\sum_{j=m^{\prime}+1}^{m} e^{-\Im w_{\jmath} x_{1}}
$$

Then we have a similar results as [5, Lemma A.3] as follows. The proof is omitted. 
Lemma 2.2. Let Assumption 2.1 be satisfied. Assume that $z_{i} \neq z_{j}$ for $1 \leq i \leq m^{\prime}$ and $m^{\prime}+1 \leq j \leq m$ and for $m^{\prime}+1 \leq i<j \leq m$. Then there exist positive numbers $C_{1}$ and $C_{2}$ such that for $k=0, \cdots, m^{\prime}-1$ the following three estimates hold:

$$
\begin{gathered}
\left|D_{1}(0, \cdots, k-1, k+1, \cdots, m-1)\left(z, w, x_{1}\right)\right| \leq C_{1} M^{\prime} \eta \\
\mid D_{2}(0, \cdots, k-1, k+1, \cdots, m-1)\left(z, w, x_{1}\right) \\
-D\left(0, \cdots, k-1, k+1, \cdots, m^{\prime}-1\right)\left(z^{\prime}, w^{\prime}, x_{1}\right)\left(t_{m^{\prime}+1} \cdots \cdot t_{m}\right)^{m^{\prime}} E(t) \mid \\
\leq \underset{\rho \in S_{2}}{C_{2} \max _{2}\left|D\left(\rho(1), \cdots, \rho\left(m^{\prime}-1\right)\right)\left(z^{\prime}, w^{\prime}, x_{1}\right)\right| \eta,} \\
-D\left(0, \cdots, k-1, k+1, \cdots, m^{\prime}-1\right)\left(z^{\prime}, w^{\prime}, x_{1}\right)\left(t_{m^{\prime}+1} \cdots t_{m}\right)^{m^{\prime}} E(t) \mid \\
\leq\left(C_{1} M^{\prime}+C_{2} \max _{\rho \in S_{2}}\left|D\left(\rho(1), \cdots, \rho\left(m^{\prime}-1\right)\right)\left(z^{\prime}, w^{\prime}, x_{1}\right)\right|\right) \eta .
\end{gathered}
$$

For $k=m^{\prime}, \cdots, m-1$, the following three estimates hold:

$$
\begin{gathered}
\left|D_{1}(0, \cdots, k-1, k+1, \cdots, m-1)\left(z, w, x_{1}\right)\right| \leq C_{1} M^{\prime} \eta^{k-m^{\prime}+1}, \\
\left|D_{2}(0, \cdots, k-1, k+1, \cdots, m-1)\left(z, w, x_{1}\right)\right| \\
\leq C_{2} \max _{\rho \in S_{2}}\left|D\left(\rho(1), \cdots, \rho\left(m^{\prime}-1\right)\right)\left(z^{\prime}, w^{\prime}, x_{1}\right)\right| \eta^{k-m^{\prime}+1}, \\
\left|D(0, \cdots, k-1, k+1, \cdots, m-1)\left(z, w, x_{1}\right)\right| \\
\leq\left(C_{1} M^{\prime}+C_{2} \max _{\rho \in S_{2}}\left|D\left(\rho(1), \cdots, \rho\left(m^{\prime}-1\right)\right)\left(z^{\prime}, w^{\prime}, x_{1}\right)\right|\right) \eta^{k-m^{\prime}+1} .
\end{gathered}
$$

The bijection $\rho$ satisfying

$$
\left\{\rho\left(m^{\prime}+1\right), \cdots, \rho(m-1)\right\}=\left\{m^{\prime}, \cdots, k-1, k+1, \cdots, m-1\right\}
$$

is uniquely determined. Call this $\rho$ as $\rho_{\max }$. Denote

$$
L^{\prime \prime}(\rho)=\left\{\begin{array}{l}
\rho\left(m^{\prime}+1\right)+\cdots+\rho(m-1)-\left(m^{\prime \prime}-1\right) m^{\prime \prime} / 2, \quad \text { for } \rho \in S_{1} \\
\rho\left(m^{\prime}\right)+\cdots+\rho(m-1)-\left(m^{\prime \prime}-1\right) m^{\prime \prime} / 2, \quad \text { for } \rho \in S_{2} .
\end{array}\right.
$$


Lemma 2.3. Let Assumption 2.1 be satisfied. Assume that $z_{i} \neq z_{j}$ for $1 \leq i \leq m^{\prime}$ and $m^{\prime}+1 \leq j \leq m$ and for $m^{\prime}+1 \leq i<j \leq m$. Then there exist a positive number $C_{1}$ such that for $k=m^{\prime}, \cdots, m-1$ and $j=1, \cdots, m^{\prime \prime}$,

$$
\begin{gathered}
\left|F_{k, j}(z)\right| \leq C_{1} \eta^{k} \\
\left|F_{k, j}(z)-f_{k, j, \rho_{\max }}(z)\right| \leq C_{1} \eta^{k+1} .
\end{gathered}
$$

Proof. Note that $C\left(\rho\left(m^{\prime}+1\right), \cdots, \rho(m-1)\right)\left(z^{\prime \prime}(j)\right)$ is a homogeneous polynomial of order $L^{\prime \prime}(\rho)+m^{\prime \prime}-1$ for $\rho \in S_{1}$. This implies that in the expression of $F_{k, j}(z)$ the largest order of $C\left(\rho\left(m^{\prime}+1\right), \cdots, \rho(m-1)\right)\left(z^{\prime \prime}(j)\right)$ is $m^{\prime} m^{\prime \prime}+m^{\prime \prime}-1-k$ and it is attained only by $\rho=\rho_{\max }$. On the other hand, for $j=1, \cdots, m^{\prime \prime}$ we have

$$
E_{j}\left(z^{\prime \prime}\right) E(z)=\eta^{m^{\prime \prime}-1+m^{\prime} m^{\prime \prime}} E_{j}\left(\eta z^{\prime \prime}\right) E(\eta z)
$$

Hence a similar method as in the proof of (2.7) can be applied to this lemma. For details we refer to [5, Lemma A.3].

\section{$\S 3 . \quad$ Proof of Theorem}

To prove Theorem we need several steps. Denote the roots of $L_{\varepsilon}(\xi)=0$ with respect to $\xi_{1}$ by $\tau_{j}\left(\varepsilon, \xi^{\prime}\right), j=1, \cdots, m$ and those of $L_{0}(\xi)=P_{2}(\xi)=0$ with respect to $\xi_{1}$ by $\sigma_{j}\left(\xi^{\prime}\right), j=1, \cdots, m^{\prime}$, respectively. It is well known that $\tau_{j}\left(\varepsilon, \xi^{\prime}\right)$, $j=1, \cdots, m$ are continuous in $\left(\varepsilon, \xi^{\prime}\right)$ for $\varepsilon \neq 0$ and $\sigma_{j}\left(\xi^{\prime}\right), j=1, \cdots, m^{\prime}$ are continuous in $\xi^{\prime}$. Put $B_{R}=\left\{\left|\xi^{\prime}\right| \leq R\right\}, p=p_{2,0} / p_{1,0}, \theta=\arg (-p), \Theta=e^{i \theta / m^{\prime \prime}}, \zeta=e^{2 \pi i / m^{\prime \prime}}$, and $\tau_{j}^{\prime}=\zeta^{j-m^{\prime}-1}, j=m^{\prime}+1, \cdots, m$.

First let us begin with the following lemma, which can be obtained through a similar argument as in [3, Lemma 2.2].

Lemma 3.1. For every positive number $R$, there exist a positive number $\varepsilon_{R}$ with $\varepsilon_{R}<1$ and continuous functions $\tau_{j, 1}\left(\varepsilon, \xi^{\prime}\right), j=1, \cdots, m$ on $\left[0, \varepsilon_{R}\right] \times B_{R}$ satisfying

$$
\limsup _{\varepsilon \downarrow 0 \xi^{\prime} \in B_{R}}\left|\tau_{j, 1}\left(\varepsilon, \xi^{\prime}\right)\right|=0, \quad j=1, \cdots, m
$$

such that for $m^{\prime}+1 \leq i<j \leq m$ and for $1 \leq i \leq m^{\prime}, m^{\prime}+1 \leq j \leq m$

$$
\tau_{i}\left(\varepsilon, \xi^{\prime}\right) \neq \tau_{j}\left(\varepsilon, \xi^{\prime}\right), \quad \text { on }\left(0, \varepsilon_{R}\right] \times B_{R}
$$


and

$$
\begin{gathered}
\tau_{j}\left(\varepsilon, \xi^{\prime}\right)=\sigma_{j}\left(\xi^{\prime}\right)+\tau_{j, 1}\left(\varepsilon, \xi^{\prime}\right), \quad \text { for } j=1, \cdots, m^{\prime} ; \\
\varepsilon^{1 / m^{\prime \prime}} \tau_{j}\left(\varepsilon, \xi^{\prime}\right)=\Theta \tau_{j}^{\prime}|p|^{1 / m^{\prime \prime}}+\varepsilon^{1 / m^{\prime \prime}} \tau_{0}\left(\xi^{\prime}\right)+\varepsilon^{1 / m^{\prime \prime}} \tau_{j, 1}\left(\varepsilon, \xi^{\prime}\right),
\end{gathered}
$$

for $j=m^{\prime}+1, \cdots, m$. Here

$$
\tau_{0}\left(\xi^{\prime}\right)=\left(p_{1,0} p_{2,1}\left(\xi^{\prime}\right)-p_{1,1}\left(\xi^{\prime}\right) p_{2,0}\right) /\left(m^{\prime \prime} p_{1,0} p_{2,0}\right)
$$

which is a polynomial of $\xi^{\prime}$ whose order is at most one.

Remark. Put $\eta=\varepsilon^{1 / m^{\prime \prime}}, \quad z=\tau$, and $t=\eta z$, then Lemma 3.1 implies Assumption 2.1 .

Put $b(\tau)=\left(\tau^{j-1} ; j \downarrow 1, \cdots, m\right)$ and $c_{j}=\left(\delta_{j, k} ; k \downarrow 1, \cdots, m\right)$, where $\delta_{j, k}$ is Kronecker's delta. Denote by $Y_{j}\left(\varepsilon, x_{1}, \xi^{\prime}\right), j=1, \cdots, m$ the fundamental solutions of the following ordinary differential equation with parameter $\left(\varepsilon, \xi^{\prime}\right)$ :

(ODE)

$$
\left\{\begin{array}{l}
L_{\varepsilon}\left(D_{1}, \xi^{\prime}\right) Y\left(\varepsilon, x_{1}, \xi^{\prime}\right)=0 ; \\
D_{1}^{k-1} Y\left(\varepsilon, 0, \xi^{\prime}\right)=\delta_{j, k}, \quad j, k=1, \cdots, m .
\end{array}\right.
$$

We shall use the following abbreviation: $\tau_{j}\left(\varepsilon, \xi^{\prime}\right)=\tau_{j}, j=1, \cdots, m$ and $\tau=\left(\tau_{1}, \cdots, \tau_{m}\right)$. Then Cramer's formula implies that if $\tau_{i} \neq \tau_{j}, 1 \leq i<j \leq m$ then

$$
\begin{gathered}
Y_{j}\left(\varepsilon, x_{1}, \xi^{\prime}\right)=\sum_{k=1}^{m} e^{i \tau_{k} x_{1}} \frac{\operatorname{det}\left(b\left(\tau_{1}\right), \cdots, b\left(\tau_{k-1}\right), c_{j}, b\left(\tau_{k+1}\right), \cdots, b\left(\tau_{m}\right)\right)}{\operatorname{det}\left(b\left(\tau_{1}\right), \cdots, b\left(\tau_{m}\right)\right)} \\
=\frac{\operatorname{det}^{t}\left({ }^{t} a(0), \cdots,{ }^{t} a(j-2),{ }^{t} e\left(\tau, x_{1}\right),{ }^{t} a(j), \cdots,{ }^{t} a(m-1)\right)}{A(0, \cdots, m-1)} \\
=(-1)^{j-1} D(0, \cdots, j-2, j, \cdots, m-1)\left(\tau, \tau, x_{1}\right), \quad j=1, \cdots, m .
\end{gathered}
$$

But the last representations remain valid without any restriction on $\tau_{j}$, $j=1, \cdots, m$. For, since $B\left(l_{1}, \cdots, l_{n-1}\right)\left(z, z, x_{1}\right)$ is an entire function of $z$ and vanishes on the zeros of irreducible polynomials $z_{j}-z_{i}, \quad(1 \leq i<j \leq n)$, $B\left(l_{1}, \cdots, l_{n-1}\right)\left(z, z, x_{1}\right)$ is divided by $A(0, \cdots, n-1)(z)$ in the ring of entire functions and consequently $D\left(l_{1}, \cdots, l_{n-1}\right)\left(z, z, x_{1}\right)$ is also an entire function.

Using Lemma 2.2 and Lemma 3.1, we can prove the following two lemmas in a similar manner as in the proof of [5, Lemma 3 and Lemma 4 . 
Lemma 3.2. Let $\varepsilon_{R}$ be the same as in Lemma 3.1.

(1) For every positive number $R$, there exists a positive number $C_{R}$ such that

$$
\sup _{0<\varepsilon \leq \varepsilon_{R}, 0 \leq x_{1} \leq T,\left|\xi^{\prime}\right| \leq R}\left|Y_{j}\left(\varepsilon, x_{1}, \xi^{\prime}\right)\right| \leq C_{R}
$$

for $j=1, \cdots, m^{\prime}$.

(2) Let either $(C 1)$ or $(C 2)$ be satisfied. Then, for every positive number $R$, there exists a positive number $C_{R}$ such that

$$
\sup _{0<\varepsilon \leq \varepsilon_{R}, 0 \leq x_{1} \leq T .\left|\xi^{\prime}\right| \leq R} \varepsilon^{-\left(j-m^{\prime}\right) / m^{\prime \prime}}\left|Y_{j}\left(\varepsilon, x_{1}, \xi^{\prime}\right)\right| \leq C_{R}
$$

$$
\text { for } j=m^{\prime}+1, \cdots, m \text {. }
$$

Denote by $y_{j}\left(x_{1}, \xi^{\prime}\right), j=1, \cdots, m^{\prime}$ the fundamental solutions of the following ordinary differential equation with parameter $\xi^{\prime}$ :

(RODE)

$$
\left\{\begin{array}{l}
L_{0}\left(D_{1}, \xi^{\prime}\right) y\left(x_{1}, \xi^{\prime}\right)=0 ; \\
D_{1}^{k-1} y\left(0, \xi^{\prime}\right)=\delta_{j, k}, \quad j, k=1, \cdots, m^{\prime} .
\end{array}\right.
$$

As we have already shown in the case of $Y\left(\varepsilon, x_{1}, \xi^{\prime}\right)$,

$$
y_{j}\left(x_{1}, \xi^{\prime}\right)=(-1)^{j-1} D(0, \cdots, j-2, j, \cdots, m-1)\left(\sigma, \sigma, x_{1}\right), \quad j=1, \cdots, m^{\prime},
$$

where $\sigma_{j}=\sigma_{j}\left(\xi^{\prime}\right), j=1, \cdots, m^{\prime}$ are the roots appearing in Lemma 3.1 and $\sigma=\left(\sigma_{1}, \cdots, \sigma_{m^{\prime}}\right)$.

\section{Lemma 3.3.}

(1) Let $\varepsilon_{R}$ be the same as in Lemma 3.1. Then

$$
Y_{j}\left(\varepsilon, x_{1}, \xi^{\prime}\right) \rightarrow y_{j}\left(x_{1}, \xi^{\prime}\right), \quad j=1, \cdots, m^{\prime}
$$

uniformly on $[0, T] \times B_{R}$ when $\varepsilon \downarrow 0$.

(2) Let $\varepsilon_{R}$ be the same as in Lemma 3.1 and either (C1) or (C2) be satisfied. Then

$$
Y_{j}\left(\varepsilon, x_{1}, \xi^{\prime}\right) \rightarrow 0, \quad j=m^{\prime}+1, \cdots, m
$$

uniformly on $[0, T] \times B_{R}$ when $\varepsilon \downarrow 0$. 


\section{Denote}

$$
M_{+}=\max \left\{\mathfrak{I}\left(\Theta \tau_{j}^{\prime}\right) ; j=m^{\prime}+1, \cdots, m\right\} ; \quad M_{-}=\min \left\{\mathfrak{I}\left(\Theta \tau_{j}^{\prime}\right) ; j=m^{\prime}+1, \cdots, m\right\} .
$$

Both the maximum and the minimum are attained by one $j$ or two $j$ 's. Denote $\boldsymbol{R}_{-}=\{x \in \boldsymbol{R} ; x<0\}$. Then the following four cases can be considered:

(1) Case 1: If $m-m^{\prime} \geq 3$ or if $m-m^{\prime}=2$ and $p /|p|=-\Theta^{2} \in C \backslash\left(R_{-} \cup\{0\}\right)$, then $M_{+}>0$ and $M_{-}<0$.

(2) Case 2: If $m-m^{\prime}=2$ and $p<0$ or if $m-m^{\prime}=1$ and $p \in \mathbb{R}$, then $M_{+}=M_{-}=0$.

(3) Case 3: If $m-m^{\prime}=1$ and $\mathfrak{I} p=\mathfrak{I}(-\Theta)>0$, then $M_{+}=M_{-}<0$.

(4) Case 4: If $m-m^{\prime}=1$ and $\Im p=\Im(-\Theta)<0$, then $M_{+}=M_{-}>0$.

The condition that $(\mathrm{C} 1)$ or $(\mathrm{C} 2)$ is satisfied corresponds to Case 2 or Case 4. Note that in either case $\Im\left(\Theta \tau_{j}^{\prime}\right) \geq 0$ holds for $j=m^{\prime}+1, \cdots, m$.

Lemma 3.4. Let $R$ and $\varepsilon_{R}$ be the same as in Lemma 3.1. Then

(1) (The case that $M_{-}$is attained by only one element of $\left\{\Im\left(\Theta \tau_{j}^{\prime}\right) ; j=m^{\prime}+1\right.$, $\cdots, m\}$, say, $\mathfrak{I}\left(\Theta \tau_{l}^{\prime}\right)$.)

$$
\begin{gathered}
\lim \sup \varepsilon^{-(m-1) / m^{\prime \prime}} \mid D_{1}(0, \cdots, m-2)\left(\tau, \tau, x_{1}\right) e^{\Im\left(\Theta \tau_{i}^{\prime}\right)(|p| / \varepsilon)^{1 / m^{\prime \prime}} x_{1}} \\
\quad-F_{m-1, l-m^{\prime}}(\tau) e^{i \tau_{l}+\Im\left(\Theta \tau_{i}^{\prime}\right)(|p| / \varepsilon)^{1 / m^{\prime \prime}} x_{1}} \mid=0
\end{gathered}
$$

where the supremum is taken for $0<\varepsilon \leq \varepsilon_{0}, \delta \leq x_{1} \leq T,\left|\xi^{\prime}\right| \leq R$ for arbitrary positive numbers $\varepsilon_{0}$ and $\delta$ with $\varepsilon_{0} \leq \varepsilon_{R}$ and $\delta \leq T$.

(2) (The case that $M_{-}$is attained by two elements of $\left\{\Im\left(\Theta \tau_{j}^{\prime}\right)\right.$; $\left.j=m^{\prime}+1, \cdots, m\right\}$. In this case we may assume that these are $\mathfrak{I}\left(\Theta \tau_{l}^{\prime}\right)$ and $\mathfrak{I}\left(\Theta \tau_{l+1}^{\prime}\right)$, and that $\mathfrak{R}\left(\Theta \tau_{l}^{\prime}\right)=-\mathfrak{R}\left(\Theta \tau_{l+1}^{\prime}\right)$.)

$$
\begin{gathered}
\limsup \varepsilon^{-(m-1) / m^{\prime \prime}} \mid D_{1}(0, \cdots, m-2)\left(\tau, \tau, x_{1}\right) e^{\Im\left(\Theta \tau_{i}^{\prime}\right)(|p| / \varepsilon)^{1 / m^{\prime \prime}} x_{1}} \\
\quad-F_{m-1, l-m^{\prime}}(\tau) e^{i \tau_{l}+\Im\left(\Theta \tau_{i}^{\prime}\right)(|p| / \varepsilon)^{1 / m^{\prime \prime}} x_{1}} \\
-F_{m-1, l+1-m^{\prime}}(\tau) e^{i \tau_{l+1}+\Im\left(\Theta \tau_{i}^{\prime}\right)(|p| / \varepsilon)^{1 / m^{\prime \prime}} x_{1}} \mid=0
\end{gathered}
$$

where the supremum is taken for $0<\varepsilon \leq \varepsilon_{0}, \delta \leq x_{1} \leq T,\left|\xi^{\prime}\right| \leq R$ for arbitrary positive numbers $\varepsilon_{0}$ and $\delta$ with $\varepsilon_{0} \leq \varepsilon_{R}$ and $\delta \leq T$. 
Proof. Put $z=w=\tau$ and $k=m-1$ in Lemma 2.1. Then

$$
D_{1}(0, \cdots, m-2)\left(\tau, \tau, x_{1}\right)=\sum_{j=1}^{m^{\prime \prime}} F_{m-1, j}(\tau) e^{i \tau_{j+m} x_{1}}
$$

Put $\eta=\varepsilon^{1 / m^{\prime \prime}}, \eta_{R}=\varepsilon_{R}^{1 / m^{\prime \prime}}$, and $t=\eta \tau$. As Assumption 2.1 is satisfied, (2.10) implies that

$$
\left|F_{m-1, j}(\tau)\right| \leq C_{1} \varepsilon^{(m-1) / m^{\prime \prime}}, \quad j=1, \cdots, m^{\prime \prime}
$$

Lemma 3.1 implies that

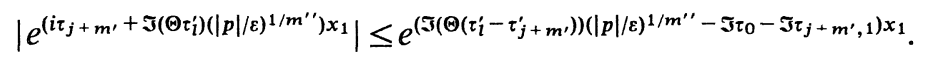

Assume that either $j+m^{\prime} \neq l$ in the case of (1) or $j+m^{\prime} \neq l, l+1$ in the case of (2). Then $\mathfrak{I}\left(\Theta\left(\tau_{l}^{\prime}-\tau_{j+m^{\prime}}^{\prime}\right)\right)<0$. Since $\left|\mathfrak{I} \tau_{0}\left(\xi^{\prime}\right)\right|$ and $\left|\Im \tau_{j+m^{\prime}, 1}\left(\varepsilon, \xi^{\prime}\right)\right|$ are bounded, we have, for $\delta \leq x_{1} \leq T$, the right-hand side of (3.9) tends to zero as $\varepsilon_{0} \downarrow 0$. Thus we come to the conclusion.

Put, for $j=1, \cdots, m^{\prime \prime}$,

$$
A_{j}=(-1)^{I\left(\rho_{\max }\right)+1+m^{\prime \prime}} /\left\{\left(\Theta|p|^{1 / m^{\prime \prime}} \zeta^{j-1}\right)^{m-1} \prod_{k=1}^{m^{\prime \prime}-1}\left(1-\zeta^{k}\right)\right\}
$$

which are non-zero constants depending only on $p$ and $j$.

Lemma 3.5. Let $R$ and $B_{R}$ be the same as in Lemma 3.1. Then

$$
\lim _{\varepsilon \downarrow 0} F_{m-1, j}(\tau) \varepsilon^{-(m-1) / m^{\prime \prime}}=A_{j}, \quad j=1, \cdots, m^{\prime \prime},
$$

uniformly on $B_{R}$.

Proof. Since

$$
C\left(m^{\prime}, \cdots, m-2\right)\left(z^{\prime \prime}(j)\right)=\left(z_{m^{\prime}+1} \cdots \cdot z_{m^{\prime}+j-1} \cdot z_{m^{\prime}+j+1} \cdots \cdot z_{m}\right)^{m^{\prime}},
$$

we find

$$
\begin{aligned}
f_{m-1, j, \rho_{\max }}(z)=(-1)^{I\left(\rho_{\max }\right)+1+j}\left(z_{m^{\prime}+1} \cdots \cdot z_{m^{\prime}+j-1} \cdot z_{m^{\prime}+j+1} \cdots \cdot z_{m}\right)^{m^{\prime}} \\
\times E_{j}\left(z^{\prime \prime}\right) E(z) .
\end{aligned}
$$


Put $\eta=\varepsilon^{1 / m^{\prime \prime}}$ and $t=\eta z$. Then

$$
\begin{gathered}
f_{m-1, j, \rho_{\max }}(z)=f_{m-1, j, \rho_{\max }}(t) \eta^{m-1} \\
=(-1)^{I\left(\rho_{\max }\right)+1+j} \eta^{m-1}\left(t_{m^{\prime}+1} \cdots \cdot t_{m^{\prime}+j-1} \cdot t_{m^{\prime}+j+1} \cdots \cdot t_{m}\right)^{m^{\prime}} E_{j}\left(t^{\prime \prime}\right) E(t) .
\end{gathered}
$$

Hence

$$
\begin{gathered}
\lim _{\eta \downarrow 0} f_{m-1, j . \rho_{\max }}(\eta \tau)\left(\Theta|p|^{1 / m^{\prime \prime}}\right)^{m-1} \\
=(-1)^{I\left(\rho_{\max }\right)+1+j}\left(\tau_{m^{\prime}+1}^{\prime} \cdots \cdots \cdot \tau_{m^{\prime}+j-1}^{\prime} \cdot \tau_{m^{\prime}+j+1}^{\prime} \cdots \cdot \tau_{m}^{\prime}\right)^{m^{\prime}} \\
\times E_{j}\left(\tau_{m^{\prime}+1}^{\prime}, \cdots, \tau_{m}^{\prime}\right) E\left(0, \cdots, 0, \tau_{m^{\prime}+1}^{\prime}, \cdots, \tau_{m}^{\prime}\right) \\
=(-1)^{I\left(\rho_{\max }\right)+1+j}\left(\tau_{m^{\prime}+1}^{\prime} \cdots \cdot \tau_{m}^{\prime}\right)^{m^{\prime}} E\left(0, \cdots, 0, \tau_{m^{\prime}+1}^{\prime}, \cdots, \tau_{m}^{\prime}\right) \\
\times\left(\tau_{m^{\prime}+j}^{\prime}\right)^{-m^{\prime}} E_{j}\left(\tau_{m^{\prime}+1}^{\prime}, \cdots, \tau_{m}^{\prime}\right) .
\end{gathered}
$$

Since $\left(\tau_{m^{\prime}+1}^{\prime} \cdots \cdot \tau_{m}^{\prime}\right)^{m^{\prime}} E\left(0, \cdots, 0, \tau_{m^{\prime}+1}^{\prime}, \cdots, \tau_{m}^{\prime}\right)=1$ and

$$
\begin{gathered}
\left(\tau_{m^{\prime}+j}^{\prime}\right)^{-m^{\prime}} E_{j}\left(\tau_{m^{\prime}+1}^{\prime}, \cdots, \tau_{m}^{\prime}\right) \\
=(-1)^{m^{\prime \prime}-j} /\left\{\left(\zeta^{j-1}\right)^{m^{\prime}+m^{\prime \prime}-1} \prod_{k=1}^{m^{\prime \prime}-1}\left(1-\zeta^{k}\right)\right\} \\
=\left(\Theta|p|^{1 / m^{\prime \prime}}\right)^{m-1} A_{j} /(-1)^{I\left(\rho_{\max }\right)+1+j},
\end{gathered}
$$

(2.11) in Lemma 2.3 implies (3.10).

Proof of Theorem. It is well known that the solution $u_{\varepsilon}(x ; \varphi)$ of $(\mathrm{CP})$ satisfies

$$
u_{\varepsilon}(x ; \varphi)=\sum_{j=1}^{m} F_{\xi^{\prime} \rightarrow x^{\prime}}^{-1}\left(Y_{j}\left(\varepsilon, x_{1}, \xi^{\prime}\right) \hat{\varphi}_{j}\left(\xi^{\prime}\right)\right)
$$

and that the solution $u_{0}\left(x ; \varphi^{\prime}\right)$ of $(\mathrm{RCP})$ satisfies

$$
u_{0}\left(x ; \varphi^{\prime}\right)=\sum_{j=1}^{m^{\prime}} F_{\xi^{\prime} \rightarrow x^{\prime}}^{-1}\left(y_{j}\left(x_{1}, \xi^{\prime}\right) \hat{\varphi}_{j}\left(\xi^{\prime}\right)\right)
$$

where ${ }^{\wedge}$ denotes the partial Fourier transformation with respect to $x^{\prime}, \xi^{\prime}$ denotes the dual variable of $x^{\prime}$, and $F_{\xi^{\prime} \rightarrow x^{\prime}}^{-1}$ denotes its inverse Fourier transformation. Put 


$$
\begin{gathered}
u_{1, \varepsilon}\left(x ;\left(\varphi^{\prime}, 0\right)\right)=\sum_{j=1}^{m^{\prime}} F_{\xi^{\prime} \rightarrow x^{\prime}}^{-1}\left(Y_{j}\left(\varepsilon, x_{1}, \xi^{\prime}\right) \hat{\varphi}_{j}\left(\xi^{\prime}\right)\right) ; \\
u_{2, \varepsilon}\left(x ;\left(0, \varphi^{\prime \prime}\right)\right)=\sum_{j=m^{\prime}+1}^{m} F_{\xi^{\prime} \rightarrow x^{\prime}}^{-1}\left(Y_{j}\left(\varepsilon, x_{1}, \xi^{\prime}\right) \hat{\varphi}_{j}\left(\xi^{\prime}\right)\right) .
\end{gathered}
$$

Then $u_{\varepsilon}(x ; \varphi)=u_{1, \varepsilon}\left(x ;\left(\varphi^{\prime}, 0\right)\right)+u_{2, \varepsilon}\left(x ;\left(0, \varphi^{\prime \prime}\right)\right)$.

First we shall show the sufficiency of (C1) or (C2) for the admissibility. Since $\varphi \in F^{-1}\left(C_{0}^{\infty}\left(\boldsymbol{R}^{n-1}\right)\right)^{m}$, there exists a positive number $R$ such that supp $\hat{\varphi}_{j} \subset$ $B_{R}, j=1, \cdots, m$. By (3.1), Lebesgue's bounded convergence theorem can be applied to (3.11) when $\varepsilon \downarrow 0$. Then (3.4) implies that

$$
\begin{gathered}
\underset{\varepsilon \downarrow 0}{\lim u_{1, \varepsilon}}\left(x ;\left(\varphi^{\prime}, 0\right)\right)=\sum_{j=1}^{m^{\prime}} F_{\xi^{\prime} \rightarrow x^{\prime}}^{-1}\left(\lim _{\varepsilon \downarrow 0} Y_{j}\left(\varepsilon, x_{1}, \xi^{\prime}\right) \hat{\varphi}_{j}\left(\xi^{\prime}\right)\right) \\
=\sum_{j=1}^{m^{\prime}} F_{\xi^{\prime} \rightarrow x^{\prime}}^{-1}\left(y_{j}\left(x_{1}, \xi^{\prime}\right) \hat{\varphi}_{j}\left(\xi^{\prime}\right)\right)=u_{0}\left(x ; \varphi^{\prime}\right),
\end{gathered}
$$

which remains true even when neither (C1) nor (C2) is satisfied. Hence (3.2) and (3.5) imply that

$$
\lim _{\varepsilon \downarrow 0} u_{2, \varepsilon}\left(x ;\left(0, \varphi^{\prime \prime}\right)\right)=\sum_{j=m^{\prime}+1}^{m} F_{\xi^{\prime} \rightarrow x^{\prime}}^{-1}\left(\lim _{\varepsilon \downarrow 0} Y_{j}\left(\varepsilon, x_{1}, \xi^{\prime}\right) \hat{\varphi}_{j}\left(\xi^{\prime}\right)\right)=0
$$

Thus $\lim _{\varepsilon \downarrow 0} u_{\varepsilon}(x ; \varphi)=u_{0}\left(x ; \varphi^{\prime}\right)$. Since this convergence remains true in $C\left(\boldsymbol{R}_{+}^{n}\right)$, (CP) are admissible.

Next we shall show the necessity of (C1) or (C2) for the admissibility. It is enough to show that if $M_{-}<0$ then there exists a Cauchy data $\tilde{\varphi}$ such that the solutions $u_{\varepsilon}(x ; \tilde{\varphi})$ diverge in $C\left(\boldsymbol{R}_{+}^{n}\right)$. Choose $\tilde{\varphi}=\left(0, \cdots, 0, \varphi_{m}\right)$, where $\varphi_{m} \neq 0$ and put

$$
v_{\varepsilon, l}\left(x ; \varphi_{m}\right)=F_{\xi^{\prime} \rightarrow x^{\prime}}^{-1}\left((-1)^{m-1} D_{l}(0, \cdots, m-2)\left(\tau, \tau, x_{1}\right) \hat{\varphi}_{m}\left(\xi^{\prime}\right)\right)
$$

for $l=1,2$. Then

$$
u_{\varepsilon}(x ; \tilde{\varphi})=F_{\xi^{\prime} \rightarrow x^{\prime}}^{-1}\left(Y_{m}\left(\varepsilon, x_{1}, \xi^{\prime}\right) \hat{\varphi}_{m}\left(\xi^{\prime}\right)\right)=v_{\varepsilon, 1}\left(x ; \varphi_{m}\right)+v_{\varepsilon, 2}\left(x ; \varphi_{m}\right) .
$$

Since (2.8) in Lemma 2.2 implies that $\lim _{\varepsilon \downarrow 0} v_{\varepsilon, 2}\left(x ; \varphi_{m}\right)=0$ in $C\left(\boldsymbol{R}_{+}^{n}\right)$, it is enough to show that $v_{\varepsilon, 1}\left(x ; \varphi_{m}\right)$ diverge when $\varepsilon \downarrow 0$. Put 


$$
\begin{aligned}
w_{\varepsilon}\left(x ; \varphi_{m}\right)= & \sum_{j=1}^{m^{\prime \prime}} F_{\xi^{\prime} \rightarrow x^{\prime}}^{-1}\left((-1)^{m-1} F_{m-1, j}(\tau) \varepsilon^{-(m-1) / m^{\prime \prime}}\right. \\
& \left.\times e^{i\left(\tau_{j}+m^{\prime}-\Theta \tau_{1}^{\prime}(|p| / \varepsilon)^{1 / m^{\prime \prime}}\right) x_{1}} \hat{\varphi}_{m}\left(\xi^{\prime}\right)\right) .
\end{aligned}
$$

Then Lemma 2.1 implies that

$$
\begin{aligned}
v_{\varepsilon, 1}\left(x ; \varphi_{m}\right) & =\sum_{j=1}^{m^{\prime \prime}} F_{\xi^{\prime} \rightarrow x^{\prime}}^{-1}\left((-1)^{m-1} F_{m-1, j}(\tau) e^{i \tau_{j+m^{\prime} x_{1}}} \hat{\varphi}_{m}\left(\xi^{\prime}\right)\right) \\
& =\varepsilon^{(m-1) / m^{\prime \prime}} e^{i \Theta \tau_{i}^{\prime}(|p| / /)^{1 / m^{\prime \prime} x_{1}}} w_{\varepsilon}\left(x ; \varphi_{m}\right) .
\end{aligned}
$$

(1) The case that $M_{-}$is attained by only one $\mathscr{F}\left(\Theta \tau_{l}^{\prime}\right)$. Lemma 3.1, 3.4, and 3.5 imply that

$$
\lim _{\varepsilon \downarrow 0} w_{\varepsilon}\left(x ; \varphi_{m}\right)=(-1)^{m-1} A_{l-m^{\prime}} F_{\xi^{\prime} \rightarrow x^{\prime}}^{-1}\left(e^{i \tau_{0}\left(\xi^{\prime}\right) x_{1}} \hat{\varphi}_{m}\left(\xi^{\prime}\right)\right),
$$

in $C\left(\boldsymbol{R}_{+}^{n}\right)$.

(2) The case that $M_{-}$is attained by two $\mathfrak{I}\left(\Theta \tau_{l}^{\prime}\right)$ and $\mathfrak{I}\left(\Theta \tau_{l+1}^{\prime}\right)$. Put $L=\mathfrak{R}\left(\Theta \tau_{l+1}^{\prime}\right)$ and $\varepsilon_{n}\left(x_{1}\right)=|p| /\left\{\pi\left(n+(m-1) / m^{\prime \prime}\right)\left(L x_{1}\right)^{-1}\right\}^{m^{\prime \prime}}$, for every integer $n$. Then $L>0, \mathfrak{R}\left(\Theta \tau_{l}^{\prime}\right)=-L$, and

$$
e^{2 i L\left(|p| / \varepsilon_{n}\left(x_{1}\right)\right)^{1 / m^{\prime \prime} x_{1}}}=\zeta^{m-1}=A_{l-m^{\prime}} / A_{l+1-m^{\prime}}
$$

Hence Lemma 3.1, 3.4, and 3.5 imply that

$$
\begin{gathered}
\lim _{n \uparrow \infty} w_{\varepsilon_{n}}\left(x ; \varphi_{m}\right)=(-1)^{m-1} F_{\xi^{\prime} \rightarrow x^{\prime}}^{-1}\left(A_{l-m^{\prime}} e^{i \tau_{0}\left(\xi^{\prime}\right) x_{1}} \hat{\varphi}_{m}\left(\xi^{\prime}\right)\right. \\
\left.+A_{l+1-m^{\prime} \zeta^{m-1}} e^{i \tau_{0}\left(\xi^{\prime}\right) x_{1}} \hat{\varphi}_{m}\left(\xi^{\prime}\right)\right) \\
=2(-1)^{m-1} A_{l-m^{\prime}} F_{\xi^{\prime} \rightarrow x^{\prime}}^{-1}\left(e^{i \tau_{0}\left(\xi^{\prime}\right) x_{1}} \hat{\varphi}_{m}\left(\xi^{\prime}\right)\right)
\end{gathered}
$$

in $C\left(\boldsymbol{R}_{+}^{n}\right)$.

In either case, $\lim _{n \uparrow \infty} w_{\varepsilon_{n}}\left(x ; \varphi_{m}\right)$ is a non-trivial real analytic function in $\boldsymbol{R}_{+}^{n}$. On the other hand, for $x_{1}>0, \varepsilon^{(m-1) / m^{\prime \prime}} e^{i \Theta \tau_{i}^{\prime}(|p| / \varepsilon)^{1 / m^{\prime \prime} x_{1}}}$ diverge when $\varepsilon \downarrow$. Thus $v_{\varepsilon_{n}, 1}\left(x ; \varphi_{m}\right)$ diverge in $C\left(\boldsymbol{R}_{+}^{n}\right)$ when $n \uparrow \infty$. 


\section{References}

[1] Ashino, R., The reducibility of the boundary conditions in the one-parameter family of elliptic linear boundary value problems I, Osaka J. Math., 25 (1988), 737-757.

[2] - On the admissibility of singular perturbations in Cauchy problems, Osaka $\mathrm{J}$. Math., 26 (1989), 387-398.

[3] The reducibility of the boundary conditions in the one-parameter family of elliptic linear boundary value problems II, Osaka J. Math., 26 (1989), 535-556.

[ 4 ] $\longrightarrow$ On the weak admissibility of singular perturbations in Cauchy problems, Publ. RIMS, Kyoto Univ., 25 (1989), 947-969.

[ 5 ] , On Nagumo's $\mathrm{H}^{s}$-stability in singular perturbations, Publ. RIMS, Kyoto Univ., 27 (1991), 551-575.

[6] Macdonald, I.G., Symmetric functions and Hall polynomials, Oxford University Press, 1979.

[7] Shilov, G.E., Linear Algebra, Dover, 1977.

[ 8 ] Uchiyama, K., $\mathrm{L}^{2}$-theory of singular perturbation of hyperbolic equations I. (A priori estimates with parameter E.), J. Fac. Sci. Univ. Tokyo, 39 (1992), 233-269.

[9] $\longrightarrow \mathrm{L}^{2}$-theory of singular perturbation of hyperbolic equations II. (Asymptotic expansions of dissipative type.), J. Fac. Sci. Univ. Tokyo, 40 (1993), 387-409. 